\title{
Femtosecond-laser inscribed double-cladding waveguides in Nd:YAG crystal: a promising prototype for integrated lasers
}

\author{
Hongliang Liu, ${ }^{1}$ Feng Chen, ${ }^{1, *}$ Javier R. Vázquez de Aldana, ${ }^{2}$ and D. Jaque ${ }^{3}$ \\ ${ }^{1}$ School of Physics, State Key Laboratory of Crystal Materials and Key Laboratory of Particle Physics and Particle Irradiation \\ (Ministry of Education), Shandong University, Jinan 250100, China \\ ${ }^{2}$ Laser Microprocessing Group, Universidad de Salamanca, Salamanca 37008, Spain \\ ${ }^{3}$ Fluorescence Imaging Group, Departamento de Física de Materiales, Facultad de Ciencias, \\ Universidad Autónoma de Madrid, Madrid 28049, Spain \\ *Corresponding author: drfchen@sdu.edu.cn
}

Received July 16, 2013; revised July 30, 2013; accepted July 30, 2013;

posted July 31, 2013 (Doc. ID 194061); published August 22, 2013

\begin{abstract}
We report on the design and implementation of a prototype of optical waveguides fabricated in Nd:YAG crystals by using femtosecond-laser irradiation. In this prototype, two concentric tubular structures with nearly circular cross sections of different diameters have been inscribed in the Nd:YAG crystals, generating double-cladding waveguides. Under $808 \mathrm{~nm}$ optical pumping, waveguide lasers have been realized in the double-cladding structures. Compared with single-cladding waveguides, the concentric tubular structures, benefiting from the large pump area of the outermost cladding, possess both superior laser performance and nearly single-mode beam profile in the inner cladding. Double-cladding waveguides of the same size were fabricated and coated by a thin optical film, and a maximum output power of $384 \mathrm{~mW}$ and a slope efficiency of $46.1 \%$ were obtained. Since the large diameters of the outer claddings are comparable with those of the optical fibers, this prototype paves a way to construct an integrated single-mode laser system with a direct fiber-waveguide configuration. (c) 2013 Optical Society of America

OCIS codes: (230.7370) Waveguides; (160.3380) Laser materials; (140.3390) Laser materials processing.

http://dx.doi.org/10.1364/OL.38.003294
\end{abstract}

Light in optical waveguides can reach high optical intensities owing to the confinement of the light field within much more compact volumes; consequently, laser oscillations in active gain waveguides may possess low lasing thresholds and comparable efficiency with respect to the bulk lasers [1,2]. In addition, a single photonic chip may be constructed in waveguide platforms to achieve multiple functions [3, $\underline{4}]$. Since 1996, femtosecond (fs) laser inscription has become a powerful technique for microstructuring of various optical materials, and a wide range of photonic applications have been realized [4-14]. These guiding structures include the single-line written waveguides (so-called type I, with positive refractive index changes in the irradiated filament) [8], double-line fabricated waveguides (so-called type II, with stress-induced guiding region between the two tracks of negative index changes) [9-14], and depressed cladding waveguides (located in the core surrounded by multiple low-index tracks) [15-18]. In Nd:YAG laser crystals, both type-II stress-induced waveguides [14] and depressed singlecladding waveguides $[17,18]$ have been successfully fabricated with fs pulses. One of the advantages of the cladding waveguides is that the large-scale cross sections match the commercially available multimode fibers (with diameters of $100-400 \mu \mathrm{m}$ ), which in principle offers an opportunity to realize efficient fiber-waveguide laser systems with low costs. However, as a drawback, the cladding waveguide lasers usually show multimodal beam properties due to the large diameters of the structures [19]. The type-II stress-induced waveguides exhibit single-mode features; however, the efficient pump of such structures relies on tightly focused beams (of diameters no more than $20 \mu \mathrm{m}$ ), which cannot be achieved directly from fiber-coupled high-power diode lasers (usually out-coupled by multimode fibers with diameters of 100-400 $\mu \mathrm{m})$ [20].

In this Letter, we propose a configuration of doublecladding waveguides containing two concentric tubes with different diameters, which is, to some extent, similar to double-cladding fibers. Furthermore, spatial distribution of the lattice residual stress (as obtained from spectral shifts), fluorescence efficiency, and fluorescence linewidth were measured in an area covering the cladding waveguide. The generated waveguide lasers are found to behave as nearly single-mode, delivering higher output powers, compared with the single-cladding waveguides.

The optically polished Nd:YAG crystal used in this work was cut into dimensions of $8 \mathrm{~mm} \times 10 \mathrm{~mm} \times$ $2 \mathrm{~mm}$. The double-cladding waveguides with circular boundaries were produced by using the laser facility of Universidad de Salamanca, Spain. Figure 1(a) shows the scheme of the fs-laser fabrication procedure in the Nd:YAG crystal. We used an amplified Ti:sapphire laser system generating linearly polarized 120 fs pulses at a central wavelength of $800 \mathrm{~nm}$ (with $1 \mathrm{kHz}$ repetition rate and $1 \mathrm{~mJ}$ maximum pulse energy). The pulse energy used to irradiate the sample was set with a calibrated neutral density filter, a half-wave plate, and a linear polarizer. The sample was placed in a computer-controlled motorized three-axes stage. The beam was focused through the largest sample surface (dimensions $8 \mathrm{~mm} \times 10 \mathrm{~mm}$ ) at a depth of $150 \mu \mathrm{m}$ through a microscope objective (Leica $40 \times$, numerical aperture NA $=0.65$ ) with a pulse energy of $0.2 \mu \mathrm{J}$. The linear and nonlinear refractive indices of YAG are $n_{0}=1.83$ and $n_{2} \approx 6 \times 10^{-16} \mathrm{~cm}^{2} / \mathrm{W}$. Based on these values the YAG threshold power for self-focusing has been estimated previously to be $0.9 \mathrm{MW}$. The laser 

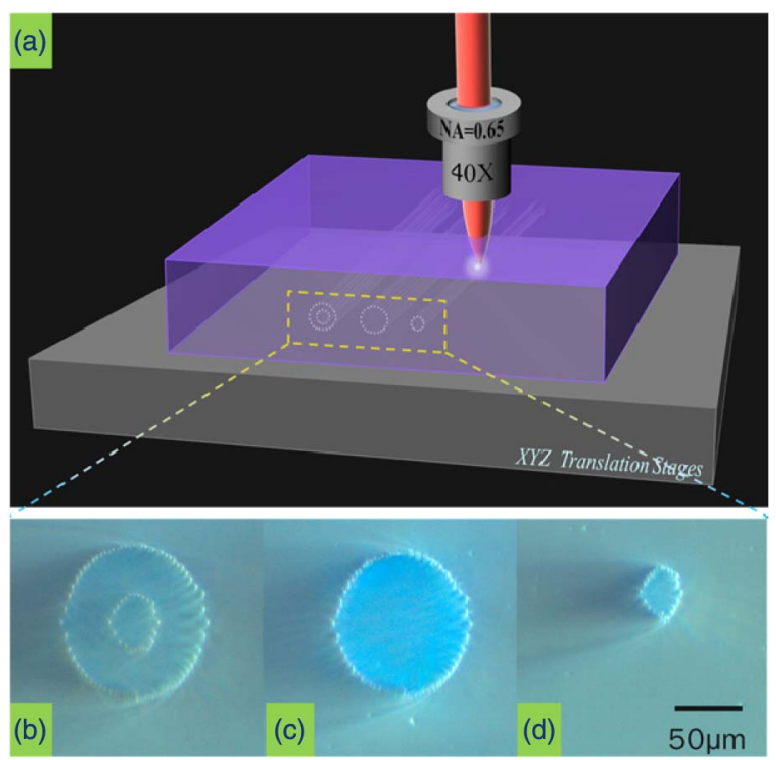

Fig. 1. (a) Fabrication schematic of fs-laser inscription process in Nd:YAG crystals, and optical images of (b) doublecladding waveguides and single-cladding waveguides with diameters of (c) 100 and (d) $30 \mu \mathrm{m}$.

power used in our experiments is estimated to be, for the pulse energy of $0.2 \mu \mathrm{J}$, close to $1.5 \mathrm{MW}$, which is above the YAG threshold power for self-focusing, and hence self-focusing is expected to occur, leading to the creation of elongated damage tracks. During the irradiation, the sample was moved at a constant speed of $700 \mu \mathrm{m} / \mathrm{s}$ in the direction perpendicular to the laser polarization and the pulse propagation, which was carefully aligned with the 10-mm-long edge of the sample, thus producing a damage track along the sample. Many parallel scans (with $\sim 3 \mu \mathrm{m}$ separation between adjacent damage tracks) were performed at different depths of the sample (from bottom to top in order to avoid the shielding of the incident pulses by the previously written damage tracks) to inscribe the double-cladding waveguide, which consisted of a tubular central structure with $30 \mu \mathrm{m}$ diameter, and concentric larger size tubular claddings $(100 \mu \mathrm{m}$ diameter). The cross section of the resulting structures in the Nd:YAG crystal can be seen in Fig. 1(b). For comparison, single-cladding waveguides with cross-section diameters of 100 and $30 \mu \mathrm{m}$ [see Figs. 1(c) and 1(d)] were produced in the same crystal. The propagation losses of the waveguides at $633 \mathrm{~nm}$ were about 1.9, 1.7, and $2.0 \mathrm{~dB} / \mathrm{cm}$ at TE polarization, respectively.

The ultrafast laser inscription produced obvious modifications of the microstructural and fluorescent properties in the filaments (fs-laser focus), as clearly illustrated in the fluorescence images included in Fig. 2 , which correspond to the concentric tubular configuration. The graphs correspond to the spatial distribution of the residual stress affecting the Nd:YAG network pressure (as obtained from the induced spectral shifts of fluorescence lines and that are given in kBar units), the FWHM (in $\mathrm{cm}^{-1}$ units), and the intensity (in a.u. units) of the ${ }^{4} \mathrm{~F}_{3 / 2} \rightarrow{ }^{4} \mathrm{I}_{9 / 2}$ inter-Stark level emission line of $\mathrm{Nd}^{3+}$ ions around $940 \mathrm{~nm}$ (this line is used for this analysis since it has been found to be hypersensitivity to slight changes in the $\mathrm{Nd}^{3+}$ environments, for example, strain, volume
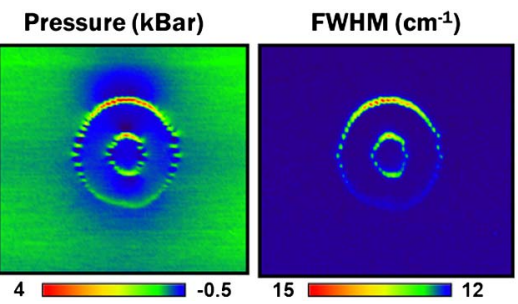

Intensity (a. u.)

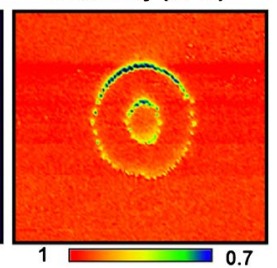

Fig. 2. Spatial distributions of (a) pressure, (b) FWHM, and (c) intensity corresponding to the ${ }^{4} \mathrm{~F}_{3 / 2} \rightarrow{ }^{4} \mathrm{I}_{9 / 2}$ inter-Stark level emission line of $\mathrm{Nd}^{3+}$ in the double-cladding waveguide.

changes, and disorder). As we can see, a compressive stress is created along the waveguide contour, being as large as $4 \mathrm{kBar}$. The spatial variation of the linewidth reveals a large broadening of the fluorescence lines at waveguide contour, which is proportional to the former one. This, indeed, could be attributed to the local disorder of the Nd:YAG system as a consequence of the damage caused by the tightly focused fs laser. Finally, the image about the fluorescence intensity (revealing a net reduction at the damage focus) unequivocally reveals the creation of defects induced at these locations (i.e., partially damaged areas). According to the previous work [21], the light is confined within the structures as a consequence of the lower barrier caused by the localized damage. Therefore, the fluorescence images included in Fig. 2 show a strong modification of the Nd: YAG system, caused by simultaneous damage, compression, and disorder, only at the waveguide's contour in the cladding waveguides. Nevertheless, the Nd:YAG network with slight modification at the waveguide volume leads to a partial dilatation that is not accompanied by any relevant disorder or damage. With the consistency of the focus, where the refractive index decreases consecutively measured about $3 \times 10^{-3}$, the concentric tubular cladding waveguide could confine the light intensively propagating in the structure.

The continuous-wave (cw) laser experiments were performed by using an end-face coupling system. A tunable cw Ti:sapphire laser (Coherent MBR 110) generated a polarized light pump beam at $808 \mathrm{~nm}$. A spherical convex lens with a focal length of $70 \mathrm{~mm}$ was used to focus the pump beam, reducing it to a diameter of $\sim 140 \mu \mathrm{m}$ and coupling it into the waveguide. To achieve the Fabry-Perot cavity for the $1.06 \mu \mathrm{m}$ laser emission in the waveguides, two dielectric mirrors were butt-coupled to the two polished end facets [the input one with a high reflectivity (>99\%) at $1.06 \mu \mathrm{m}$ and high transmission (98\%) at $808 \mathrm{~nm}$ and the output one with reflectivity of $60 \%$ at $1.06 \mu \mathrm{m}$ and $>99 \%$ at $808 \mathrm{~nm}$. The generated waveguide lasers were collected with a $20 \times$ microscope objective lens (NA $=0.4)$ and imaged by an IR CCD camera through an aperture. A spectrometer was used to analyze the emission spectra of the generated laser beam. In the laser experiments, the double-cladding waveguides showed stable and remarkable performance.

The near-field intensity profiles of the output lasers from the double-cladding waveguide and single-cladding waveguides at $1064 \mathrm{~nm}$ are depicted in Figs. 3(d)-3(f). For comparison, Figs. 3(a)-3(c) show the multimode near-field intensity distribution at $632.8 \mathrm{~nm}$ from the 

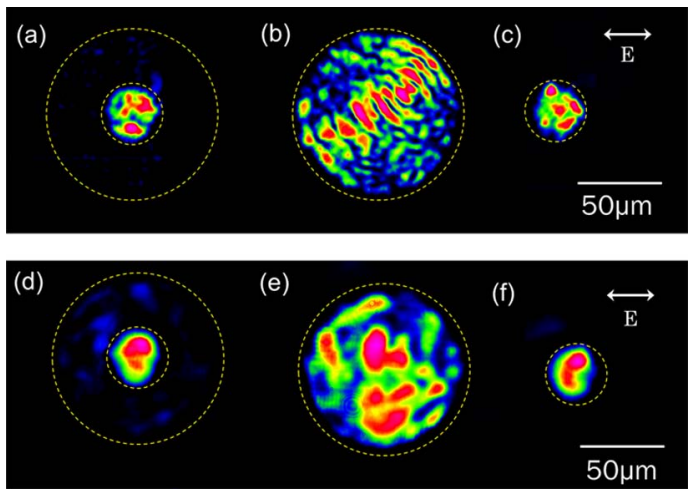

Fig. 3. Near-field intensity profiles of the TE mode of the laser for the waveguides at (a)-(c) $632.8 \mathrm{~nm}$ and (d)-(f) $1064 \mathrm{~nm}$.

inner core of the double-cladding waveguide and the single-cladding waveguides with diameters of 100 and $30 \mu \mathrm{m}$, respectively. As one can see, the $30-\mu \mathrm{m}$-diameter cladding structures, i.e., in both the inner core of the double cladding and that of the single-cladding [see Figs. 3(d) and 3(f)] supported single-mode waveguide lasers, which is significantly different from the larger-scale claddings [see Fig. 3(e)]. It should be pointed out that, different from the type-II Nd:YAG waveguides [14], the laser performances as well as the guidance features from these cladding waveguides are similar for both TE and TM polarizations, which shows good flexibility for a normal diode laser pump.

Figure $\underline{4}$ shows the output power of generated lasers at $1064 \mathrm{~nm}$ as a function of absorbed pump power at $808 \mathrm{~nm}$ obtained from the inner $30-\mu \mathrm{m}$-diameter core of the double-cladding waveguides and the single-cladding waveguides with diameters of 100 and $30 \mu \mathrm{m}$, respectively. The inset graph shows the spectrum of the laser oscillation at $1064 \mathrm{~nm}$ corresponding to the ${ }^{4} \mathrm{~F}_{3 / 2} \rightarrow{ }^{4} \mathrm{I}_{11 / 2}$ emission line of the $\mathrm{Nd}^{3+}$ ions, with the FWHM being $0.5 \mathrm{~nm}$. From the linear fits of the output power as a function of the absorbed power, a slope efficiency of $38.9 \%$ can be obtained from the inner core of double-cladding waveguides. In addition, the obtained maximum output power of the $1064 \mathrm{~nm}$ lasers was $102 \mathrm{~mW}$ from the

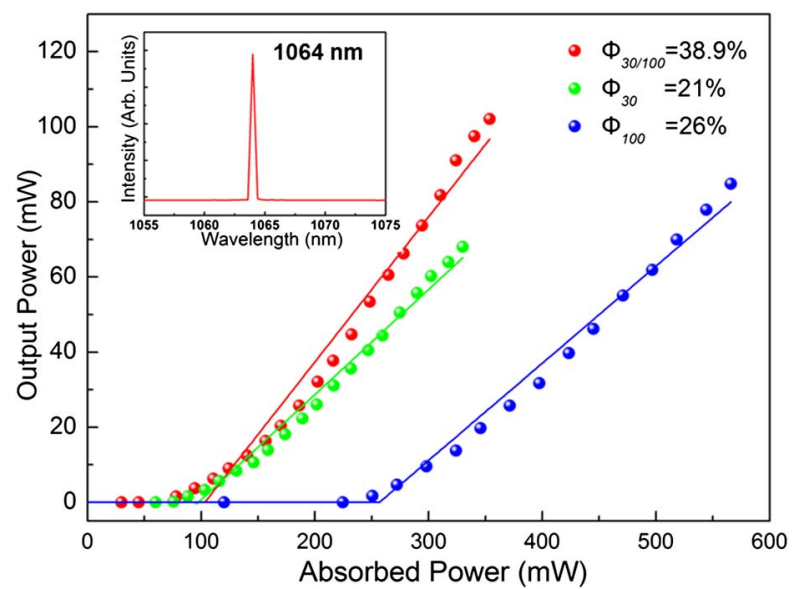

Fig. 4. Output power of the generated lasers at $1064 \mathrm{~nm}$ as a function of the absorbed pump power at $808 \mathrm{~nm}$. The inset graph shows the spectrum of the laser at $1064 \mathrm{~nm}(0.5 \mathrm{~nm}$ FWHM). double-cladding (inner mode) waveguide when the absorbed power was $350 \mathrm{~mW}$. And the lasing thresholds were determined to be $103 \mathrm{~mW}$. For a good comparison, the laser curves of the single-cladding structures were depicted in the figure. We obtained waveguide lasers with slope efficiencies of $26 \%$ and $21 \%$ for the single-cladding structures with diameters of 100 and $30 \mu \mathrm{m}$, respectively. These values are considerably lower than those from the double-cladding waveguide core. Moreover, the lasing thresholds of the single claddings with a diameter of $100 \mu \mathrm{m}$ were $256 \mathrm{~mW}$, which was higher than that of the double-cladding core. More importantly, the maximum output powers of the $30 \mu \mathrm{m}$ nearly single-mode waveguide lasers from the double-cladding cores are as high as $102 \mathrm{~mW}$, which is $50 \%$ higher than that of the $30 \mu \mathrm{m}$ single-cladding structure $(68 \mathrm{~mW})$ with similar threshold. This owes to the large-area pump but compressed output volume, which means that more pumping light goes into the whole structure but only generates guided IR lasers in the inner core region. This advantage of the double-cladding structures reflects on the laser performances, resulting in reduced lasing thresholds, higher slope efficiencies, and increased output powers.

Concentric larger size tubular claddings with diameter of $100 \mu \mathrm{m}$ and an inner waveguide core with diameter of $30 \mu \mathrm{m}$ were fabricated with a pulse energy of $0.42 \mu \mathrm{J}$. A thin film with a high reflectivity at $1.06 \mu \mathrm{m}$ and high transmission at $808 \mathrm{~nm}$ was coated (serving as the input mirror) on the sample to form the laser resonance cavity with the end-face of the crystal. The laser experiment was performed under the same conditions without using an output mirror at room temperature. From the linear fit of the data shown in Fig. 5, the maximum output power obtained are 346 and $384 \mathrm{~mW}$ for the waveguide laser at TM and TE polarization, respectively. The inset graph shows the generated single mode of the laser supported both at TE and TM polarization. The slope efficiencies are $41.5 \%$ and $46.1 \%$, respectively, which are quite higher than those obtained for the sample without thin film. Meanwhile, from comparison of the laser performance as depicted in Table 1 , this experiment certified that the effect of using thin film to form the laser resonant

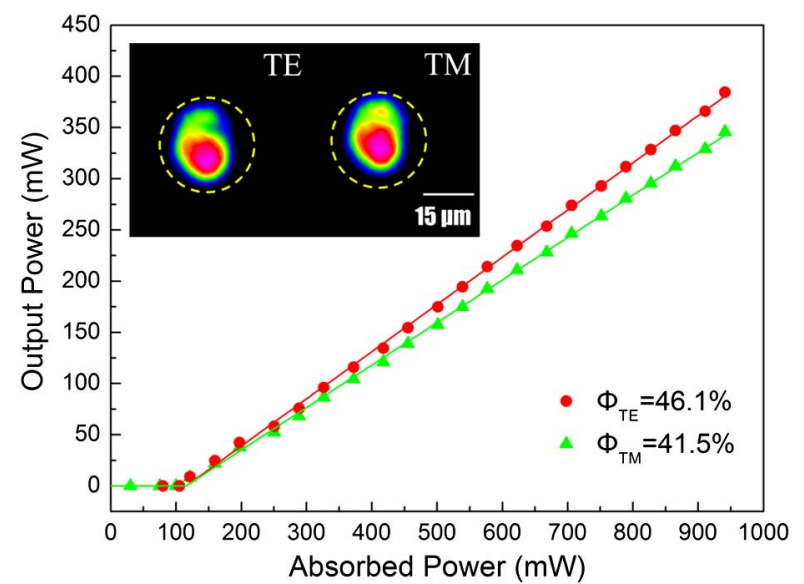

Fig. 5. Output power of the laser as a function of the absorbed power at room temperature. The inset graph displays the laser mode of the laser from the waveguide cores at both TE and TM polarization. 
Table 1. Characteristics of Double-Cladding Waveguides

\begin{tabular}{lcccc}
\hline Polarization Direction & Mirror/Film & Output Power (Max.) $(\mathrm{mW})$ & Slope Efficiency (\%) & Lasing Threshold (mW) \\
\hline TE & Mirror & 102 & 38.9 & 101 \\
TM & Mirror & 91 & 32 & 104 \\
TE & Film & 384 & 46.1 & 106 \\
TM & Film & 346 & 41.5 & 105 \\
\hline
\end{tabular}

cavity is much more efficient than using additional mirrors, with an improvement of the optical conversion efficiency from $28.6 \%$ to $41 \%$ at TE mode.

In conclusion, we have fabricated concentric tubular waveguides with double claddings in Nd:YAG crystal by using fs-laser inscription. Nearly single-mode waveguide lasers with slope efficiency of $46.1 \%$ and maximum output power of $384 \mathrm{~mW}$ from the inner cores have shown superior laser performance to the standard single-cladding waveguides, which benefits from the large-area pump of the outermost cladding. It should also be pointed out that the laser performances of the cladding waveguides in the present work are limited by the powers of the pumping lasers. A few watts output power of the generated waveguide lasers could be expected in the case of an efficient pump from a fibercoupled diode laser. In addition, since one of the major advantages of the fs-laser writing technique is the capability of realizing arbitrary shapes, it is intriguing to produce more complicated geometry (e.g., D-shaped cladding with off-centered core) to improve the lasing performances. This work paves a new way toward constructing an integrated high-power, single-mode laser system with a direct fiber-waveguide configuration.

This work was supported by the National Natural Science Foundation of China (Grant No. 11274203) and the Spanish Ministerio de Ciencia e Innovación (MICINN) through Consolider Program SAUUL CSD2007-00013 and project FIS2009-09522. Support from the Centro de Láseres-Pulsados (CLPU) is also acknowledged.

\section{References}

1. C. Grivas, Prog. Quantum Electron. 35, 159 (2011).

2. F. Chen, Laser Photon. Rev. 6, 622 (2012).

3. W. Sohler, H. Hu, R. Ricken, V. Quiring, C. Vannahme, H. Herrmann, D. Büchter, S. Reza, W. Grundkötter, S. Orlov, H. Suche, R. Nouroozi, and Y. H. Min, Opt. Photon. News 19(1), 24 (2008).
4. J. Thomas, M. Heinrich, P. Zeil, V. Hilbert, K. Rademaker, R. Riedel, S. Ringleb, C. Dubs, J.-P. Ruske, S. Nolte, and A. Tünnermann, Phys. Status Solidi A 208, 276 (2011).

5. K. M. Davis, K. Miura, N. Sugimoto, and K. Hirao, Opt. Lett. 21, 1729 (1996).

6. M. Ams, G. D. Marshall, P. Dekker, J. Piper, and M. Withford, Laser Photon. Rev. 3, 535 (2009).

7. R. R. Gattass and E. Mazur, Nat. Photonics 2, 219 (2008).

8. J. Burghoff, S. Nolte, and A. Tunnermann, Appl. Phys. A 89, 127 (2007).

9. G. A. Torchia, P. F. Meilan, A. Rodenas, D. Jaque, C. Mendez, and L. Roso, Opt. Express 15, 13266 (2007).

10. Y. Tan, F. Chen, J. R. Vázquez de Aldana, G. A. Torchia, A. Benayas, and D. Jaque, Appl. Phys. Lett. 97, 031119 (2010).

11. C. Zhang, N. Dong, J. Yang, F. Chen, J. R. Vázquez de Aldana, and Q. Lu, Opt. Express 19, 12503 (2011).

12. T. Calmano, J. Siebenmorgen, F. Reichert, M. Fechner, A. Paschke, N. Hansen, K. Petermann, and G. Huber, Opt. Lett. 36, 4620 (2011).

13. C. Grivas, C. Corbari, G. Brambilla, and P. Lagoudakis, Opt. Lett. 37, 4630 (2012).

14. T. Calmano, J. Siebenmorgen, O. Hellmig, K. Petermann, and G. Huber, Appl. Phys. B 100, 131 (2010).

15. D. G. Lancaster, S. Gross, H. Ebendorff, K. Kuan, M. Ams, and M. Withford, Opt. Lett. 36, 1587 (2011).

16. D. G. Lancaster, H. Ebendorff, A. Fuerbach, M. J. Withford, and T. M. Monro, Opt. Lett. 37, 996 (2012).

17. A. Okhrimchuk, V. Mezentsev, A. Shestakov, and I. Bennion, Opt. Express 20, 3832 (2012).

18. H. Liu, Y. Jia, J. R. Vázquez de Aldana, D. Jaque, and F. Chen, Opt. Express 20, 18620 (2012).

19. R. Ramponi, R. Osellame, and M. Marangoni, Rev. Sci. Instrum. 73, 1117 (2002).

20. F. Chen and J. R. Vázquez de Aldana, "Optical waveguides in crystalline dielectric materials produced by femtosecond-laser micromachining," Laser Photon. Rev., doi:10.1002/lpor.201300025 (2013).

21. A. Ródenas, G. A. Torchia, G. Lifante, E. Cantelar, J. Lamela, F. Jaque, L. Roso, and D. Jaque, Appl. Phys. B 95, 85 (2009). 